\title{
Adaptive Acoustical-Environmental Assessment for the Focused Acoustic Field-05 At-sea Exercise
}

\author{
Ding Wang*, Pierre F. J. Lermusiaux ${ }^{\dagger}$, Patrick J. Haley ${ }^{\dagger}$, Wayne G. Leslie ${ }^{\dagger}$, and Henrik Schmidt ${ }^{*}$ \\ ${ }^{*}$ Department of Mechanical Engineering, Massachusetts Institute of Technology, Cambridge, MA 02139 \\ ${ }^{\dagger}$ Division of Engineering and Applied Sciences, Harvard University, Cambridge, MA 02138
}

\begin{abstract}
Variabilities in the coastal ocean environment span a wide range of spatial and temporal scales. From an acoustic viewpoint, the limited oceanographic measurements and today's ocean modeling capabilities can't always provide oceanic-acoustic predictions in sufficient detail and with enough accuracy. Adaptive Rapid Environmental Assessment (AREA) is a new adaptive sampling concept being developed in connection with the emergence of the Autonomous Ocean Sampling Network (AOSN) technology. By adaptively and optimally deploying in-situ measurement resources and assimilating these data in coupled nested ocean and acoustic models, AREA can dramatically improve the ocean estimation that matters for acoustic predictions and so be essential for such predictions. These concepts are outlined and preliminary methods are developed and illustrated based on the Focused Acoustic Forecasting-05 (FAF05) exercise. During FAF05, AREA simulations were run in real-time and engineering tests carried out, within the context of an at-sea experiment with Autonomous Underwater Vehicles (AUV) in the northern Tyrrhenian sea, on the eastern side of the Corsican channel.
\end{abstract}

\section{INTRODUCTION}

In coastal regions, wind driven flows, tidal currents, river outflows, internal waves, solitary waves, fronts, eddies, thermal changes etc are some of the commonly dominant oceanographic processes. These processes make the coastal oceanacoustic environment highly variable in time and space [1]-[8]. In the water column, the temperature profile, salinity profile, plankton distribution profile etc can vary in complex dynamical ways, driven by the variety of coastal oceanographic processes and their coupling. Current flows also strongly interact with the littoral bottom topography which can be highly variable. In the seabed, bathymetric profiles vary in time and space too, which in turn make the dynamics of the water column extremely complex. The properties of the seabed are also variable, which impact acoustic predictions.

Variabilities in the coastal ocean environment span a wide range of spatial and temporal scales [7], [9]. Conventional oceanographic measurement can not provide the ability to synoptically observe all those dynamically interlocking, patchy and intermittent processes in coastal ocean, especially for sub-meso-scales short in time and space [10]. Consequently the coastal environment will be always under-sampled at these small and fast scales. Oceanographic forecasting by modeling and data assimilation such as the Harvard Ocean Prediction System with Error Subspace Statistical Estimation (HOPS/ESSE) can produce 4-D oceanographic field estimates and their associated uncertainties [11], [12]. However, the spatial and temporal grids used in computation are limited by the available computational resources, and the initial conditions can be relatively unknown due to the environmental undersampling [13]. So, even using nested computational grids, spatial scale smaller than hundred meters in the horizontal, and meters in the vertical cannot be modeled deterministically over large coastal regions (see Fig. 1).

Modern modeling and assimilation frameworks have a capability of representing the smaller, sub-grid-scale variability statistically [13], [14]. From an acoustic viewpoint, very small scale variabilities are averaged out by the acoustic wave length; while the sub-meso scale variabilities of the order of the acoustic wavelength make the coastal ocean acoustic environment largely unknown with many uncertainties in terms of imperfect sound velocity, depth of the thermocline etc. Such uncertainties can be responsible for a large part of the acoustic prediction uncertainty [1], [15]. The uncertainty of the acoustic predictability is critical to the dB-budget of classical sonar systems by directly affecting the detection and false alarm probabilities. It is also one of the major obstacles to adapting new model-based sonar processing frameworks, such as matched field processing (MFP) [16], to the coastal environment. The acoustic uncertainty associated with the spatially and temporarily varying sound speed and the random characteristics of the bottom are also of critical influence to acoustic communication systems, which with the integration of new Autonomous Ocean Sampling Network (AOSN) [17] concept in the operational Navy are becoming of increasing tactical significance.

To determine the environmental variability of the critical sub-meso scales and short temporal scales, a rapid in-situ measurement capability has long been recognized as a tactical need [1]. However, its implementation is being constrained by limited resources. Consequently, the limited availability of high-resolution in-situ measurement data for assimilation into the modeling framework may severely limit the usefulness of the forecasts to the acoustic environment prediction. Acknowledging that the size of the ocean area relevant to an acoustic problem is usually as large as tens of kilometers, the acoustic-purposed coastal environmental assessment is facing the classical conflict between resolution, needed to capture the fine scale variability and coverage, needed for the large scale environmental phenomena. Thus, the Rapid Environmental Assessment (REA) resources available must focus on the environmental uncertainties critical to the specific acoustic system. A quantitative and adaptive approach is necessary. 


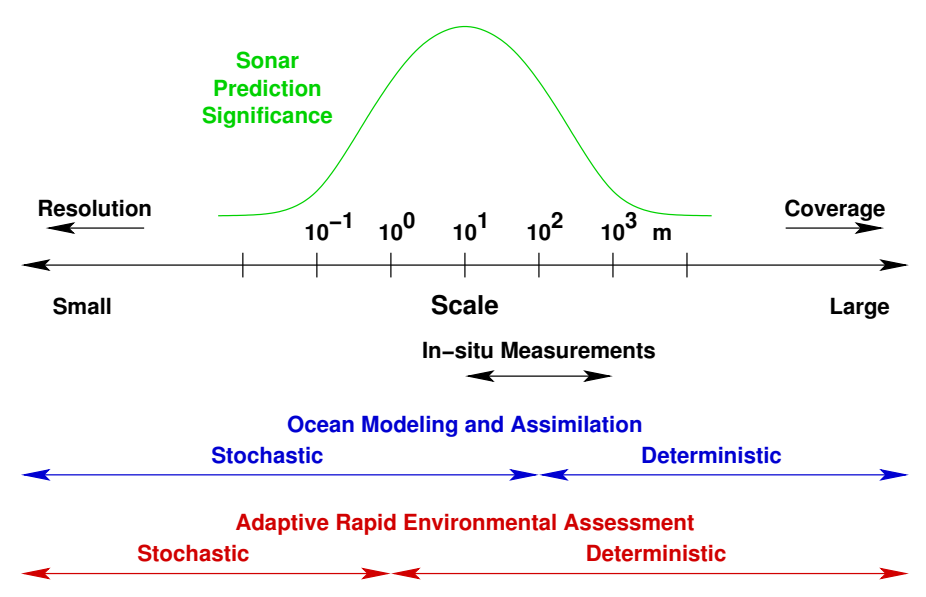

Fig. 1. Multi-scale environmental assessment. The typical sonar systems performance is dependent on acoustic environment variability over a wide range of scales. Optimal environmental assessment will therefore be a compromise between conflicting requirements of coverage and resolution. By targeting areas of high sensitivity to the sonar system through in-situ measurements, the deterministic assessment range will be shifted towards smaller scales.

Adaptive Rapid Environmental Assessment (AREA) — a new adaptive acoustical-environmental sampling approach based on coupled oceanic-acoustic forecasts is currently being developed in connection with the emergence of the new Autonomous Ocean Sampling Network (AOSN) technology [18]. In principle AREA is a probabilistic approach to the adaptive sampling problem of littoral REA and envisioned as a real time tactical tool for not only capturing, but also minimizing the acoustic uncertainty of significance to specific sonar systems. In AREA, with ocean forecasting providing large-scale coverage, identifying regions and features with strong uncertainty such as coastal fronts, the limited high-resolution tactical resources can be deployed in a manner which is optimal to the acoustic forecasting [11], [19]. Consequently, the limit of deterministic characterization may be shifted significantly towards smaller scales; a much finer resolution can be obtained in the ocean forecasting without sacrificing coverage and this will make the acoustic forecasting uncertainty minimized (see Fig. 1).

The AREA framework can also be used to minimize oceanic uncertainties, biological uncertainties etc [19], [20], or to objectively evaluate the performance of new REA concepts, such as Acoustically Focused Ocean Sampling (AFOS) [18] and Acoustic Data Assimilation (ADA) [8], [21].

\section{AREA}

Fig. 2 shows the structure of the AREA system and connections with ocean environmental models. Via data assimilation, the HOPS/ESSE and the Geographic \& Geological modeling produce an ensemble of environmental realizations for the water column and the seabed respectively. The acoustic measurement and inversion methods can be utilized to improve the ocean environmental predictions and uncertainties. The quantitative uncertainty-maps provide guidance for locating large uncertainties and so guide the sampling plans that are computed by AREA. Compared with sound velocity in the water column, the variabilities in bathymetry are less rapid and can be captured by a side- scan/sub-bottom profiling AUV,

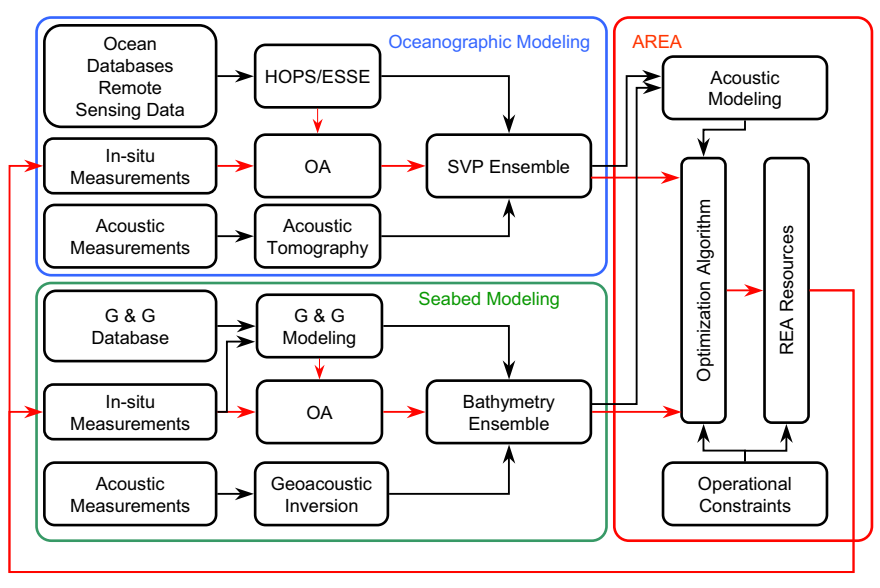

Fig. 2. AREA wiring diagram. Fore- and now-casts of the local oceanography and geology are producing spatial and temporal environmental statistics in the form of realization ensembles. These ensembles are then used as input to environmental acoustic models to provide associated realizations for the sonar performance, e.g. in the form of probability of detection and false alarms. To minimize the uncertainty of the acoustic prediction and therefore improve the probability of detection to false alarm ratio, the realization ensemble of oceanacoustic environment and the operational constraints are used to determine an optimal deployment strategy for the REA resources. The REA data are then objective analyzed based on the forecast spatial scales. The resulting reduced uncertainty now-casts are then used for the acoustic prediction.

water depth detection etc. Thus, AREA presently focuses more on the water column and treats bathymetry deterministically. In the acoustic modeling, the Range-dependent Acoustic Model (RAM PE Code) - a popular wave-theory technique for solving range-dependent propagation problems in the ocean is used [22]. By coupling the ocean, seabed and acoustic models, acoustic prediction uncertainties can be generated via Monte Carlo simulations. The weighted sum of the acoustic prediction uncertainties is the objective function in the AREA optimization algorithm which aims to select the sampling plan that reduces these integrated predicted uncertainties. This optimization is an important focus of the present manuscript.

Under operational REA, the optimization generates an optimal plan for allocating the REA resources, such as an optimal AUV path, in real-time. Thereafter, REA resources are deployed according to this optimal plan and in-situ measurement data focusing on the most critical uncertainties are collected and passed back to the oceanographic model and seabed model in a short time. Those new local data are rapidly assimilated in the models [8], [11], and ocean environmental and acoustic predictions for the next day are generated. This is the Daily AREA Cycle, which updates the optimal REA resources allocation pattern everyday.

This Daily AREA Cycle constitutes a first level of adaptivity in AREA. In addition to the static optimal REA, the optimization problem can be treated as a Sequential Decision Making Problem and modeled in the Dynamic Programming framework, in which the REA resources allocation pattern is not predetermined but generated on-board. An optimal adaptive sampling strategy is then produced, as a function of the data sampled by the autonomous data-collecting platforms, instead of a predetermined optimal sampling pattern. As indicated by 
the red lines in Fig. 2, the dynamic optimization algorithm only outputs the optimal sampling pattern for the next step; after the local data in the next step is collected and rapidly mapped by objective analysis or assimilated in real-time, a new ocean prediction is computed to optimize the subsequent sampling pattern. The whole optimal REA resources allocation pattern is adaptively generated step by step on-board. This is the second level of adaptivity.

This second level of adaptivity in AREA involves dynamic programming (DP). However, it is known that a DP problem is usually $\mathcal{N} \mathcal{P}$ hard [23]. Determining an optimization approach for the adaptive sampling strategy that can be computed on-board can thus be extremely difficult. However in some particular cases, this difficult problem can be solved by indirect methods (see Section III).

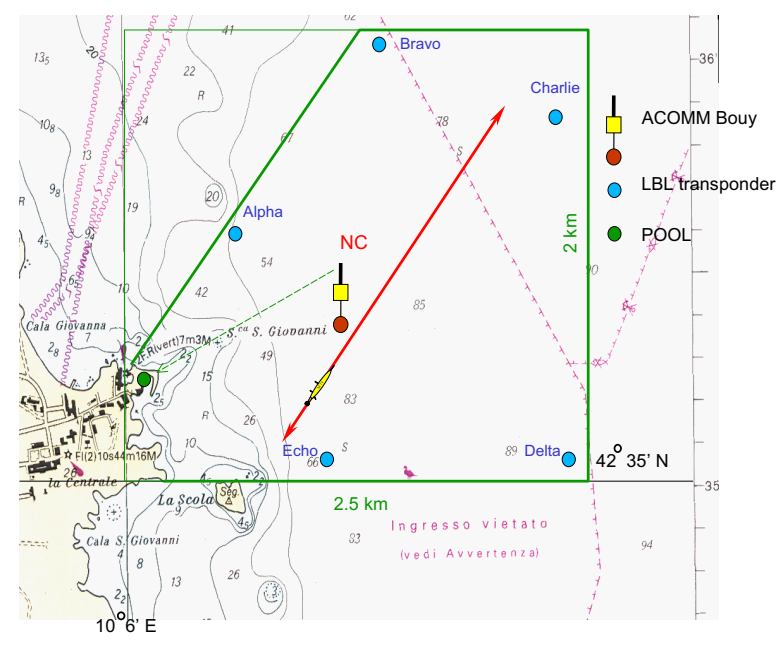

Fig. 3. FAF05 experiment site, Pianosa, Italy.

An integrated Adaptive Rapid Environmental Assessment Simulation Framework (AREASF) is created to search for the (sub)-optimal sampling patterns and/or sampling strategies, and test the optimization effects before costly on-site experiments [19]. The AREASF can also be used to observe how AREA will work and test if real-time adaptive sampling is feasible. Some new AOSN techniques such as Autonomous Sensor Platforms, MOOS-IvP Autonomy Architecture etc have been recently developed at MIT [24]. From 7/17/2005 to $7 / 26 / 2005$, the Focused Acoustic Forecasting-05 (FAF05) experiment was held off Pianosa, Italy (Fig. 3), within the northern Tyrrhenian sea, on the eastern side of the Corsican channel. The AREA concept was tested for two weeks within the AREASF, including connections to ocean and seabed models. AOSN techniques were tested at sea but the complete AREA framework was only carried out in simulations, based on real ocean data collected at sea within the AOSN exercises.

\section{FAF05 REAL-TIME AREA SIMULATIONS}

The Real-time AREA Simulations in FAF05 were initiated for developing methodologies and engineering tests [25]. The objectives included:

- Develop new algorithms and software for initiating the coupling of the Harvard real-time ocean environmental modeling, uncertainty prediction and adaptive sampling methodologies with the MIT AREA and acoustic predictions schemes.

- Develop optimization algorithms and software for generating (sub)-optimal adaptive sampling strategies.

- Test and improve those algorithms and softwares in realtime.

- Issue physical-acoustical adaptive sampling recommendations every day based on 1-to-2 days environmental forecasts of fields and uncertainties.

The vertical slice along the red arrow line shown in Fig. 3 was the main experiment area for the real-time AREA simulations. A CTD (Conductivity-Temperature-Depth) carried by an AUV was the REA source.

\section{A. Ocean-Acoustic Modeling}

a) Ocean Modeling: HOPS was run daily for 13 days, forced by a combination of Aladin atmospheric forcing (from DHMZ Croatia) and FNMOC atmospheric forcing. The ocean model of HOPS was set-up in stand-alone, one-way- and two-way- nested modeling configurations, in 2 domains: (i) a high-resolution mini-HOPS domain along the eastern coast of Pianosa (the region of MIT-FAF05 operations) and (ii) a coarser resolution domain south of Elba and east of Pianosa (see Fig. 4). The model resolutions and domain sizes are given in TABLE I. The $100 \mathrm{~m}$ resolution is designed to capture some sub-mesoscale dynamics relevant to acoustic propagation.

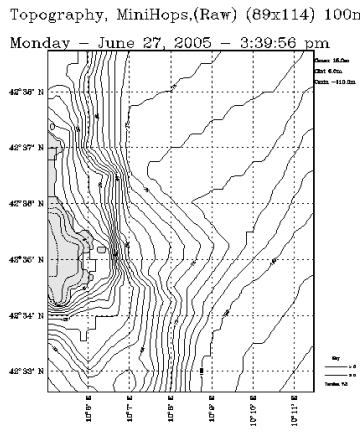

(a)

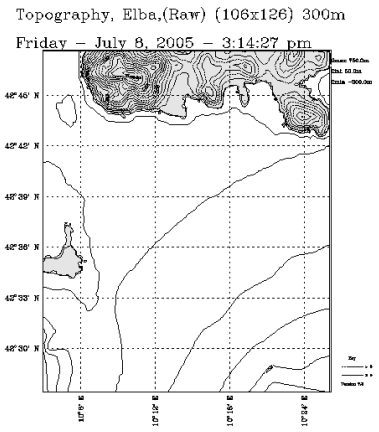

(b)
Fig. 4. HOPS 2-way nested domains: a) Pianosa domain, b) Elba domain.

$$
\text { TABLE I }
$$

TWO-WAY NESTED OCEAN DOMAIN RESOLUTION AND SIZES.

\begin{tabular}{|c|c|c|c|}
\hline \multicolumn{2}{|c|}{} & Mini-HOPS & Elba \\
\hline \multicolumn{2}{|c|}{ Resolution } & $100 \mathrm{~m}$ & $300 \mathrm{~m}$ \\
\hline \multirow{2}{*}{ Size } & $n x \times n y \times n z$ & $89 \times 114 \times 21$ & $106 \times 126 \times 21$ \\
\cline { 2 - 4 } & Extent & $8.8 \times 11.3 \mathrm{~km}$ & $31.5 \times 37.5 \mathrm{~km}$ \\
\hline
\end{tabular}

Fig. 5 illustrates the HOPS daily simulations and forecasts of the ocean environment. The ocean model assimilated the satellite sea surface temperature (SST) snapshots as available. The 1-day forecasts of the temperature and current fields at $20 \mathrm{~m}$ depths (near the main depth of the thermocline) are shown in the Pianosa (Fig. 5(a) 5(b)) and Elba (Fig. 5(c) 5(d)) domains. Effects on the circulation of the two islands and of the horizontal temperature gradients are clearly visible. 


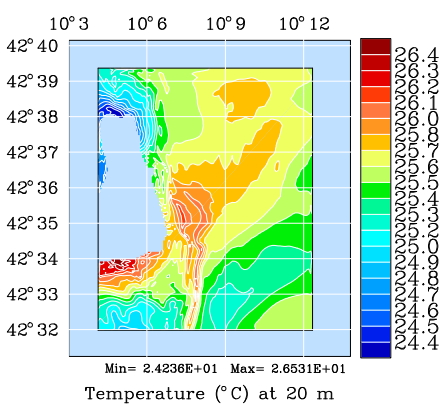

(a)

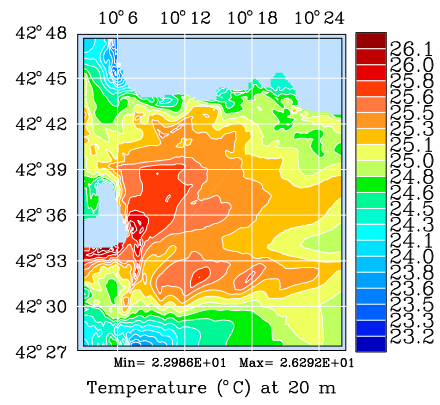

(c)

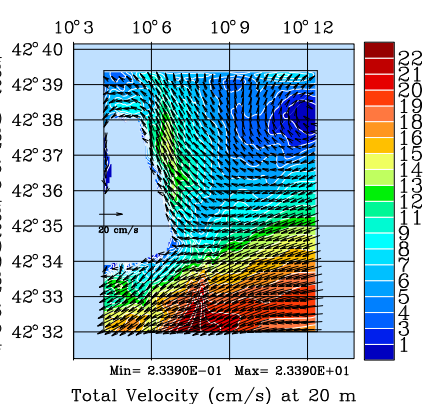

(b)

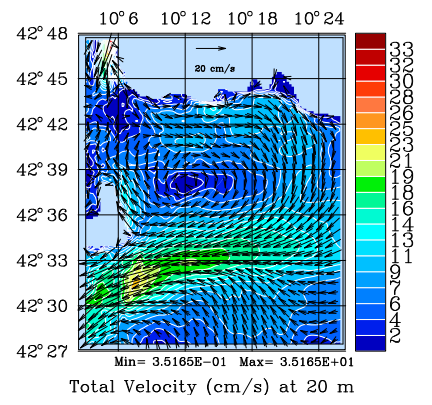

(d)
Fig. 5. HOPS 2-way nested forecasts for the morning of July 25, 2005 (local time), issued on July 24. (a) and (b): Temperature and total velocity overlaid with current vectors at $20 \mathrm{~m}$ depth in the Pianosa domain; (c) and (d): as (a) and (b) for the Elba domain.

b) Acoustic Modeling: It was assumed that a $100 \mathrm{~Hz}$ continuous-wave sound source was located at $r=1950 \mathrm{~m}$, $z=35 \mathrm{~m}$ and the transmission loss (TL) at $5 \mathrm{~m}$ depth was chosen as the acoustic signal which mattered in the AREA objective (see Fig. 6). To implement acoustic simulations and optimization in real-time, the RAM code was carefully configured so that TLs could be computed as fast as possible with sufficient precision. For coupled ocean-acoustic computations, a preliminary data-transformation interface was created to connect the HOPS/ESSE output with the RAM code, and so allow rapid and RAM-compatible extraction of the acousticrelated data from the HOPS/ESSE output. After setting up the HOPS/ESSE oceanographic model, seabed model, RAM code and coupling them together, a pipeline was created with sound velocity profile (SVP) and SST data as input, TLs at the receiver's depth as output.

\section{B. Yoyo Control}

At the site of the FAF05 experiment, the depth of the thermocline often leads to the main SVP uncertainties. Therefore, the adaptive sampling strategy that aims to capture the vertical variability of the thermocline due to fronts, eddies, internal waves, etc. can often capture the dominant SVP feature and its uncertainties and so also minimize the TL uncertainty. To track the vertical variability of the thermocline, a thermoclineoriented AUV path control was researched, by which an AUV can be given guidance about the depths of the thermocline and move around these depths. Since the thermocline is the region where the sound speed changes rapidly with depth, a simple criterion determining the relative position between the AUV and the thermocline is to compare the absolute value of local vertical gradient of sound speed $\left|\frac{\partial c}{\partial z}\right|$ with a threshold. By doing so, the AUV can estimate whether it is above, inside of or below the thermocline.

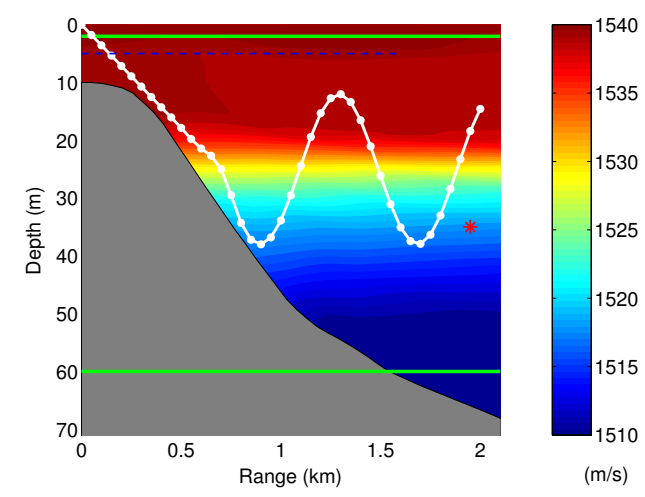

Fig. 6. Illustration of the acoustic configuration (source: red star. Target TL depth: blue dashed line) and of the thermocline-oriented AUV yoyo track.

It is assumed that at the beginning of the mission, the AUV stays on the surface. While it is diving, its CTD collects data every second. The $\left|\frac{\partial c}{\partial z}\right|$ is estimated via Linear Least Squares Fitting method based on every $p$ CTD data. If at the beginning, $\left|\frac{\partial c}{\partial z}\right| \leq \gamma$, where $\gamma$ is the threshold, and then $\left|\frac{\partial c}{\partial z}\right|$ becomes greater than $\gamma$, and after that $\left|\frac{\partial c}{\partial z}\right|$ becomes lower than $\gamma$ again, then the criterion will indicate that the AUV is now below the thermocline and it will turn around upwards. Thereafter while the AUV is going up, if $\left|\frac{\partial c}{\partial z}\right|$ becomes greater than and then lower than $\gamma$ again, the criterion indicates that the AUV is now above the thermocline and it will turn around downwards. An upper bound and a lower bound on the depth range of the AUV were also set up. Should the AUV have crossed the thermocline or not, once the lower bound or upper bound is reached, the AUV has to turn around to avoid reaching too deep depths or getting off the surface. This path control will lead the AUV to carry an up-and-down yoyo track (Fig. 6).

\section{Optimization}

In the AUV yoyo control, there are two parameters to be optimized: $p$ and $\gamma . p$ is the number of sampling points used to compute $\left|\frac{\partial c}{\partial z}\right| ; \gamma$ is the threshold used to compare with $\left|\frac{\partial c}{\partial z}\right|$. The $\gamma$ defines how rapidly the sound speed changes with depth can be linked to the thermocline. The control parameters optimization problem can be formulated as:

$$
\begin{array}{ll}
\min & f(p, \gamma) \\
\text { s.t. } & \gamma \geq 0, p \text { is a positive integer, }
\end{array}
$$

where the objective function

$$
f(p, \gamma)=E\left\{\operatorname{tr}\left(\operatorname{var}\left(T L^{O A}\right)\right)\right\}
$$

$T L^{O A}$ is the stochastic-deterministic TL vector associated with the posterior ocean estimation and its error field, after applying the yoyo control in the principal ocean prediction and assimilating the in-situ measurements via objective analysis. 
$\operatorname{var}\left(T L^{O A}\right)$ is the covariance matrix of $T L^{O A}$. Since the CTD noise may influence the estimation of the thermocline depth and thus change the AUV track, an expectation over all possible CTD noise is needed.

This optimization problem is essentially a mixed-integer non-linear programming problem. The objective function is only defined on integer-valued $p$, so it can't be solved by relaxation. Additional real-time challenges arise because of the time required to compute the objective function via Monte Carlo simulations prior to resolving this optimization problem.

In FAF05, a small size enumeration method was implemented. By repeated runs of AREASF, the objective function was computed every day for 7 potential yoyo control parameter pairs listed below, which were carefully selected. The one associated with the minimum objective function value was selected as the optimal yoyo control parameter set for the next day.

\begin{tabular}{|c|c|c|c|c|c|c|c|}
\hline & 1 & 2 & 3 & 4 & 5 & 6 & 7 \\
\hline$p$ & 20 & 20 & 20 & 30 & 30 & 30 & 30 \\
\hline$\gamma$ & 0.1 & 0.5 & 1 & 0.1 & 0.5 & 1 & 1000 \\
\hline
\end{tabular}

\section{Daily Protocol}

The real-time AREA simulations were implemented from $7 / 17 / 2005$ to $7 / 26 / 2005$. Each day, the ocean-acoustic environmental fields were predicted by HOPS/ESSE with the associated uncertainties. The data used for initialization and assimilation were satellite sea surface temperature snapshots and historical profiles of ocean temperature and salinity. To estimate ocean uncertainties, various scenarios were computed daily as a function of different initial condition estimates, assimilation procedures, modeling domains, numerical/physical model parameters and time of day. The various sound speed field predictions (in time and 3D space) were interpolated along several characteristic vertical sections and used for acoustic predictions with RAM. The ensemble of sound-speed sections and the corresponding ensemble of acoustic transmission loss fields were utilized as input to an optimization algorithm that estimated the optimal parameters of the AUV sampling pattern's for the next day(s). These optimal sampling parameter estimates and the corresponding ocean and acoustic predictions were emailed daily to the FAF05-MIT team atsea aboard the R/V Leonardo. They provided the basis for coupled physical-acoustical adaptive sampling by the MIT AUVs, aiming for optimal surveillance in the region.

The daily operational optimization procedure was:

1) The HOPS/ESSE generates 1-to-2 days environmental forecasts of fields and uncertainties.

2) Couple these forecasts with AREASF.

3) Implement the AUV yoyo control with the $i$ th parameters pair in AREASF. Output objective function value.

4) Repeat step 3 for $m$ times $(m=10)$. Calculate the average objective function value.

5) If $i<7$, then $i=i+1$ and go to step 3; otherwise, find the parameters pair associated with the minimum average objective function value and plot AUV track.

\section{E. Some Results}

A typical daily result is shown in Fig. 7, which corresponds to forecasts for the morning of $7 / 23 / 2005$. In this case, $p=$ $30, \gamma=1000$ were the optimal parameters. Analysis on the AUV yoyo control shows that when $p$ becomes larger, the thermocline-oriented criterion is be less sensitive to the CTD noise; while $\gamma$ becomes larger, the thermocline is estimated to be weaker by the criterion. If $\gamma$ is very large such as $\gamma=1000$, no thermocline is found and the AUV simply goes up-anddown between the upper and lower bound. In contrast, if $p$ and $\gamma$ become smaller, the criterion is more sensitive and thus the AUV's track is a more complex zigzag.

In FAF05, the horizontal oceanic correlation length was set to $L r=1$ or $2 \mathrm{~km}$ and the vertical correlation length to $L z=$ $5 \mathrm{~m}$. Since the $L r$ can be almost as long as the total horizontal span of experiment area, a few sampling points per depth can dramatically reduce the SVP uncertainties at that depth for a relatively large range. If the AUV can explore the deepest depths, it can very likely capture most of the SVP and TL uncertainties. On many days, the choice of bounding values for $p$ and $\gamma$, i.e. $p=30, \gamma=1000$, which make the AUV go up-and-down between the upper and low bound gave the optimal results. However, if the experiment area had spanned an area larger than $L r$, the choice $p=30, \gamma=1000$ will not often be the optimal solution.

Fig. 8 shows some interesting phenomena on $7 / 21$. For both the morning and afternoon forecasts, $p=30, \gamma=0.1$ was the optimal parameter pair. In this case, the AUV didn't go from upper to lower bound, but focused on tracking the vertical variabilities of the sound velocity. In the morning, the optimized AUV captured the main thermocline along its path, back and forth. However, in the afternoon, the AUV automatically aimed to capture the so-called 'afternoon effect' on the surface thermocline, i.e. the warming of the upper ocean layers due to the strong day-light sun, when it came back.

\section{CONCLUSION}

The principle of AREA and its preliminary computational structure were described in this paper. New algorithms and software for initiating the coupling of the HOPS/ESSE with AREASF were initiated. An adaptive sampling strategy and the associated optimization algorithm and software were developed. In FAF05, those algorithms and softwares were tested in real-time for 2 weeks. Physical-acoustical adaptive sampling recommendations based on 1-to-2 days environmental forecasts of fields and uncertainties were issued everyday. The associated analysis of the results were explained and the outcomes were encouraging.

\section{ACKNOWLEDGMENT}

This research is sponsored by the Office of Naval Research under the Capturing Uncertainty DRI, the UPS PLUSNet program, and the Adaptive Sampling and Prediction MURI. We thank Prof. Allan Robinson for inputs and support. We are grateful to E. Coelho, E. Nacini and A. Cavanna from NURC for the satellite and Alliance in-situ data, Martina Tudor from 


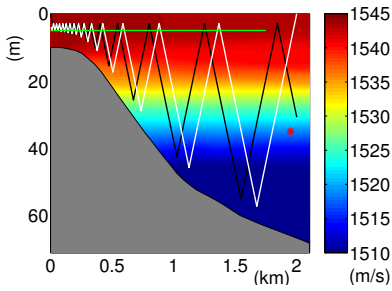

(a) Principal Estimate For SVP

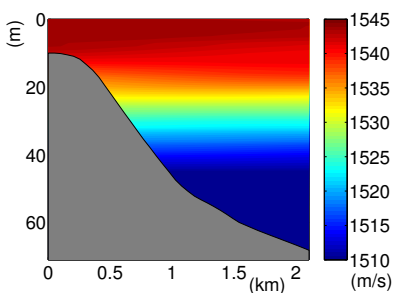

(c) Posterior Estimate For SVP

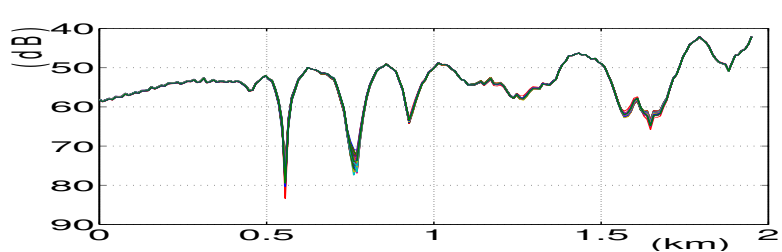

(e) Posterior TL Uncertainty

Fig. 7. AREA-HOPS-ESSE simulations for 7/23/2005. (a) Estimate of SVP output from HOPS/ESSE. Black line: forward AUV yoyo track. White line: backward track. A $100 \mathrm{~Hz} \mathrm{CW}$ sound source is located at the red point $r=$ $1950 \mathrm{~m}, z=35 \mathrm{~m}$. Green line: TL depth which is $5 \mathrm{~m}$. (b) Associated error standard deviation. (c) Posterior SVP estimate after objective analysis. (d) Associated posterior error standard deviation. (e) TL realizations associated with the environment shown in (c) and (d), where in the seabed, the sound speed is $c=1700 \mathrm{~m} / \mathrm{s}$, the density is $\rho=1700 \mathrm{~kg} / \mathrm{m}^{3}$.

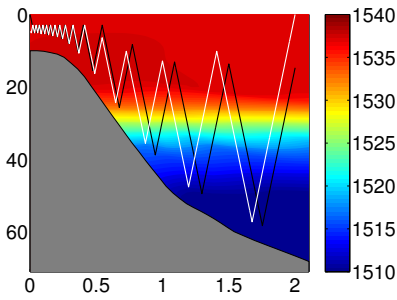

(a) Morning

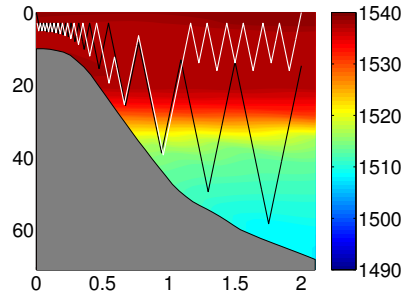

(b) Afternoon
Fig. 8. AREA simulations for $7 / 21 / 2005$. In the afternoon, when the AUV came back, it tried to capture the 'afternoon effect'.

the Cro. Met. Service for the Aladin atmospheric forcing and FNMOC for their real-time atmospheric forcing. We thank Dr. Mitchell N. Shipley (ARL Penn State) and Dr. Emanuel Coelho for their comments and suggestions.

\section{REFERENCES}

[1] H. Schmidt, "AREA: Adaptive Rapid Environmental Assessment," pp. $587-594$, in [15].

[2] E. Coelho, "Mesoscale - small scale oceanic variability effects on underwater acoustic signal propagation," pp. 49-54, in [15].

[3] S. Finette, T. Evans, and C. Shen, "Sub-mesoscale modeling of environmental variability in a shelf-slope region and the effect on acoustic fluctuations," pp. 401-408, in [15].
[4] T. F. Duda, "Relative influences of various environmental factors on $50-1000 \mathrm{~Hz}$ sound propagatioin shelf and slope areas," pp. 393-400, in [15].

[5] A. Tolstoy, S. Jesus, and O. Rodríguez, "Tidal effects on MFP via the INTIMATE96 test," pp. 457-464, in [15].

[6] T. Akal, "Effects of environmental variability on acoustic propagation loss in shallow water," pp. 229-236, in [15].

[7] P. F. J. Lermusiaux, C.-S. Chiu, and A. R. Robinson, "Modeling Uncertainties in the Prediction of the Acoustic Wavefield in a Shelfbreak Environment," in Refereed invited Manuscript, Proc. of the 5th International conference on theoretical and computational acoustics, E.C. Shang, Q. Li, and T. F. Gao, Eds. World Scientific Publishing Co., May 2001, pp. 191-200.

[8] P. F. J. Lermusiaux and C.-S. Chiu, "Four-dimensional data assimilation for coupled physical-acoustical fields," pp. 417-424, in [15].

[9] A. R. Robinson, P. Abbot, P. F. J. Lermusiaux, and L. Dillman, "Transfer of uncertainties through physical-acoustical-sonar end-to-end systems: A conceptual basis," pp. 603-610, in [15].

[10] N. M. Patrikalakis, J. J. McCarthy, A. R. Robinson, H. Schmidt, C. Evangelinos, P. Haley, S. Lalis, P. F. J. Lermusiaux, R. Tian, W. G. Leslie, and W. Cho, "Towards a Dynamic Data-Driven System for Rapid Adaptive Interdisciplinary Ocean Forecasting," in Refereed invited manuscript in Dynamic Data-Driven Application Systems, F. Darema, Ed. Kluwer Acad. Pub., Amsterdam, 2006, under review.

[11] A. R. Robinson, P. F. J. Lermusiaux, and N. Q. Sloan, "Data assimilation," The Sea: The Global Coastal Ocean, vol. 10, pp. 541-594, 1998.

[12] A. R. Robinson, "Forecasting and simulating coastal ocean processes and variabilities with the Harvard Ocean Prediction system," in Coastal Ocean Prediction, ser. AGU Coastal and Estuarine Studies Series. AGU, 1999, pp. 77-100.

[13] P. F. J. Lermusiaux, C.-S. Chiu, G. G. Gawarkiewicz, P. Abbot, A. R.Robinson, R. N. Miller, P. J. Haley, W. G. Leslie, S. J. Majumdar, A. Pang, and F. Lekien, "Quantifying Uncertainties in Ocean Predictions," Oceanography, vol. 19, pp. 92-105, March 2006.

[14] T. R. Chen, P. Ratilal, and N. C. Makris, "Mean and variance of the forward field propagated through three-dimensional random internal waves in a continental-shelf waveguide," J. Acoust. Soc. Am., vol. 118, pp. 3532-3559, 2005.

[15] N. G. Pace and F. B. Jensen, Eds., Impact of Littoral Environmental Variability on Acoustic Predictions and Sonar Performance. Kluwer Acad. Pub., 2002.

[16] A. Baggeroer, W. Kuperman, and H. Schmidt, "Matched field processing: Source localization in correlated noise as an optimum parameter estimation problem," J. Acoust. Soc. Am., vol. 83, pp. 571-587, 1988.

[17] T. Curtin, J. Bellingham, J. Catipovic, and D. Webb, "Autonomous oceanographic sampling networks," Oceanography, vol. 6, no. 3, pp. 86-94, 1993.

[18] H. Schmidt, J. G. Bellingham, and P. Elisseef, "Acoustically focused oceanographic sampling in coastal environments," in Rapid Environmental Assessment. SACLANTCEN Conference Proc. Series CP-44, E. Pouliquen, A. D. Kirwan, and R. T. Pearson, Eds., 1997.

[19] D. Wang, "Adaptive Rapid Environmental Assessment System Simulation Framework," Master Thesis, Massachusetts Institute of Technology, December 2004

[20] N. C. Makris, P. Ratilal, D. T. Symonds, S. Jagannathan, S. Lee, and R. W. Nero, "Fish Population and Behavior Revealed by Instantaneous Continental Shelf-Scale Imaging ," Science, vol. 311, pp. 660-663, Feburary 2006.

[21] P. Elisseeff, H. Schmidt, and W. Xu, "Ocean acoustic tomography as a data assimilation problem," IEEE Journal of Oceanic Engineering, vol. 27 , no. 2 , pp. $275-282,2002$.

[22] F. Jensen, W. Kuperman, M. Porter, and H. Schmidt, Computational Ocean Acoustics. American Institute of Physics, 1994.

[23] D. P. Bertsekas, Dynamic Programming and Optimal Control, 2nd ed. Athena Scientific, 2001, vol. 1.

[24] D. P. Eickstedt, M. R. Benjamin, H. Schmidt, and J. J. Leonard, "Adaptive Control in Heterogeneous Marine Sensor Platforms in an Autonomous Sensor Network," in Proceedings of the 2006 IEEE/RSJ Int. Conf. on Intelligent Robots and Systems, Oct. 2006, in Press.

[25] http://people.deas.harvard.edu/ leslie/FAF05/index.html. 